\title{
RANDOMNESS, NONLOCALITY AND INFORMATION IN ENTANGLED CORRELATIONS
}

\author{
Krzysztof Wódkiewicz* \\ Center for Advanced Studies and Department of Physics and Astronomy, \\ University of New Mexico, Albuquerque, New Mexico 87131, USA \\ and \\ Instytut Fizyki Teoretycznej, Uniwersytet Warszawski Warszawa 00-681, Hoża 69, Poland*
}

(September 30, 2018)

\begin{abstract}
It is shown that the Einstein, Podolsky and Rosen (EPR) correlations for arbitrary spin-s and the Greenberger, Horne and Zeilinger (GHZ) correlations for three particles can be described by nonlocal joint and conditional quantum probabilities. The nonlocality of these probabilities makes the Bell's inequalities void. A description that exhibits the relation between the randomness and the nonlocality of entangled correlations is introduced. Entangled EPR and GHZ correlations are studied using the Gibbs-Shannon entropy. The nonlocal character of the EPR correlations is tested using the information Bell's inequalities. Relations between the randomness, the nonlocality and the entropic information for the EPR and the GHZ correlations are established and discussed.
\end{abstract}

PACS numbers: 03.65.Bz, 89.70.+c 


\section{INTRODUCTION}

One of the most fundamental features of quantum correlations is the nonlocal and the random character of microscopic single events. Nonlocal randomness differs in a fundamental way from the randomness associated with the concept of local realism which is based on the assumption that microscopic physical systems can be described by local objective properties that are independent from observation. Local realism versus the nonlocal character of

quantum mechanics [1] has been described best in the framework of spin- $\frac{1}{2}$ EPR correlations [2]. The classical limit of quantum nonlocality has been investigated in the framework of the generalized EPR entangled correlations involving arbitrary spin- $s$ [3]. In the limit of large spin these correlations should exhibit a smooth transition to the classical regime if the measurements do not resolve the quantum properties of the spin [曰, 5].

Recently it has been shown by Greenberger, Horne and Zeilinger (GHZ) [6] that special entangled states involving three or four particles lead to a much stronger refutation of local realism. It has been shown that in many-particle correlations only a single set of observations is required in order to demolish the local reality assumption [7].

It is the purpose of this paper to show that the EPR spin- $s$ correlations and that the three-particle GHZ correlations can be visualized as nonlocal correlations between sequences of completely random numbers "0" and "1" corresponding to "no" and "yes" outcomes of the spin polarization analyzers. The coincidences between these random numbers are described by conditional probabilities which are nonlocal, i.e., are dependent on the orientations of the spin analyzers. The nonlocality of these conditional probabilities makes the Bell's inequality void, because for local realism the existence of a universal, polarization independent transition probability between the random sequences is essential.

A description that exhibits the relation between the randomness and the nonlocality of entangled quantum states is well suited to study the EPR and the GHZ correlations using the Gibbs-Shannon entropy. For a system involving two of three correlated subsystems, the information entropy can offer a measure of quantum correlation if the informations of the 
subsystems are compared with the total information of the composed system. The nonlocal character of the EPR and the GHZ correlations of the subsystems will be tested using the information Bell's inequalities involving entropies of the probabilities that can be determined from the statistics of random "yes" and "no" answers. In such a framework the relations between the randomness, the nonlocality and the entropic information for the EPR and the GHZ correlations will be established.

This paper is organized in the following way. In Sec. II general properties of the EPR correlations for arbitrary spin- $s$ are reviewed and the randomness and the nonlocality of these correlations are discussed in the framework of the quantum bivalued distribution functions. The randomness and the information of these EPR correlations is than discussed in the framework of the information entropy. The information Bell's inequality for the EPR correlations involving arbitrary spin-s is investigated. In Sec. III the random and the nonlocal properties of the GHZ correlations are discussed. The randomness and the information of these GHZ correlations are discussed in the framework of the information entropy. The corresponding information Bell's inequality for the three particles GHZ correlations is introduced and investigated.

\section{ENTANGLED EPR CORRELATIONS FOR ARBITRARY SPIN- $S$}

\section{A. RANDOMNESS AND NONLOCALITY}

The EPR entangled state of the two particles $a$ and $b$, each with spin $s$ is given by the following singlet state of the two spins:

$$
\left|\psi_{\mathrm{EPR}}\right\rangle=\sum_{m=-s}^{s} \frac{(-1)^{s+m}}{\sqrt{2 s+1}}|m ;-m\rangle .
$$

where the states $\left|m_{a} ; m_{b}\right\rangle$ correspond to the eigenvalues of the $z$-components of the two spins. In quantum mechanics, the joint spin transmission probability of detection of such a entangled state by two linear polarizers, is defined by the following formula:

$$
p(\vec{a} ; \vec{b})=\left\langle\psi_{\mathrm{EPR}}|\hat{P}(\vec{a}) \otimes \hat{P}(\vec{b})| \psi_{\mathrm{EPR}}\right\rangle
$$


In this expression $\hat{P}(\vec{a})$ and $\hat{P}(\vec{b})$ are the spin projection operators of the particles $a$ and $b$ along the polarization directions $\vec{a}$ and $\vec{b}$.

Following the basic ideas of a theory based on local reality, the transmissions of an arbitrary spin-s through the linear polarizers $\vec{a}$ and $\vec{b}$ are described by objective realities represented by normalized transmission functions $0 \leq t\left(\vec{a}, \lambda_{a}\right) \leq 1$ and $0 \leq t\left(\vec{b}, \lambda_{b}\right) \leq 1$, with hidden variables $\lambda_{a}$ and $\lambda_{b}$. In a theory based on local hidden variables (LHV), these transmission functions are local realities that are averaged over the hidden variables during a detection process. In the framework of such LHV theories the joint spin transmission is given by the following statistical average of local realities:

$$
p(\vec{a} ; \vec{b})=\int d \lambda_{a} \int d \lambda_{b} P\left(\lambda_{a} ; \lambda_{b}\right) t\left(\vec{a}, \lambda_{a}\right) t\left(\vec{b}, \lambda_{b}\right)
$$

where the hidden parameters are randomly distributed with a positive normalized distribution function:

$$
\int d \lambda_{a} \int d \lambda_{b} P\left(\lambda_{a} ; \lambda_{b}\right)=1, \quad P\left(\lambda_{a} ; \lambda_{b}\right) \geq 0
$$

This distribution function is local i.e., it is independent on the polarization directions $\vec{a}$ and $\vec{b}$. For such a local distribution of random variables, the LHV transmission probability (3) evaluated for four different polarization axes $\vec{a}, \vec{a}^{\prime}, \vec{b}, \overrightarrow{b^{\prime}}$ is restricted by the Bell's inequality 8]:

$$
-1 \leq p(\vec{a} ; \vec{b})+p\left(\vec{a}^{\prime} ; \vec{b}\right)-p\left(\vec{a} ; \vec{b}^{\prime}\right)+p\left(\vec{a}^{\prime} ; \vec{b}^{\prime}\right)-p(\vec{a})-p(\vec{b}) \leq 0
$$

The original Bell's inequalities have been derived only for spin- $\frac{1}{2}$, but it has been shown that the inequality in the form (5) is valid for correlations involving an arbitrary spin- $s$ [9].

We begin the discussion of the randomness and the nonlocality in quantum mechanics using the two-point distribution that has been introduced and applied to the discussion of quantum jumps in optical transitions [10]. Using the spectral decomposition of the spin projector operator: $\hat{P}=\int d \lambda \delta(\lambda-\hat{P})$ we rewrite the quantum mechanical joint-probability (2) in the following form: 


$$
p(\vec{a} ; \vec{b})=\int d \lambda_{a} \int d \lambda_{b} P\left(\vec{a} \lambda_{a} ; \vec{b} \lambda_{b}\right) \lambda_{a} \lambda_{b}
$$

where the distribution function is given by the following quantum mechanical average:

$$
P\left(\vec{a} \lambda_{a} ; \vec{b} \lambda_{b}\right)=\left\langle\psi_{\mathrm{EPR}}\left|\delta\left(\lambda_{a}-\hat{P}(\vec{a})\right) \otimes \delta\left(\lambda_{b}-\hat{P}(\vec{b})\right)\right| \psi_{\mathrm{EPR}}\right\rangle
$$

The projection operators $\hat{P}(\vec{a})=|\vec{a}\rangle\langle\vec{a}|$ and $\hat{P}(\vec{b})=|\vec{b}\rangle\langle\vec{b}|$ corresponding to the two orientations of the linear polarizers $\vec{a}$ and $\vec{b}$ are obtained by a rotation of the maximum "down" spin states $\left|m_{a}=-s\right\rangle$ and $\left|m_{b}=-s\right\rangle$ by angles $\tau_{a}=\frac{1}{2} \theta_{a} e^{-i \phi_{a}}$ and $\tau_{b}=\frac{1}{2} \theta_{b} e^{-i \phi_{b}}$ respectively:

$$
|\vec{a}\rangle=\exp \left(\tau_{a} \hat{S}_{a+}-\tau_{a}^{*} \hat{S}_{a-}\right)|-s\rangle \quad \text { and } \quad|\vec{b}\rangle=\exp \left(\tau_{b} \hat{S}_{b+}-\tau_{b}^{*} \hat{S}_{b-}\right)|-s\rangle
$$

where $\hat{S}_{a \pm}$ and $\hat{S}_{b \pm}$ are the spin-s ladder operators for the particle $a$ and $b$.

With the help of the distribution function (7) we have rewritten the quantum mechanical joint probability function (2) in a form given by (6) which has remarkable similarities to the LHV correlation function given by (3). Because the projection operators can have their eigenvalues equal to 1 or 0 , i.e., can represent only "yes" or "no" answers, the values $\lambda_{a}$ and $\lambda_{b}$ can take only values equal to 1 and 0 corresponding to "clicks" at the detectors. The bivalued distribution given by (7) is positive everywhere, but depends on the polarization directions $\vec{a}$ and $\vec{b}$. The distribution function which depends on the orientation $\vec{a}$ of the first analyzer and on the orientation $\vec{b}$ of the second (possibly even remote) analyzer is nonlocal. In the framework of EPR correlations, it is customary to call an analyzer-dependent distribution function a nonlocal distribution function. The nonlocality of this distribution function makes the Bell's inequality (5) void, because in order to obtain this inequality the existence of a universal, local (polarization independent) distribution in the parameters $\lambda_{a}$ and $\lambda_{b}$ (hidden parameters in this case) is essential.

From quantum mechanics we obtain that EPR correlations can be described by a distribution function of the form given by Eq. (7) with the condition that the statistical distribution of the parameters $\lambda_{a}$ and $\lambda_{b}$ is nonlocal i.e., is dependent on the polarization direction.

In order to elucidate the statistical and the random character of the nonlocal distribution 
function further, we shall perform a Bayesian analysis of the joint correlation. The joint distribution can be written in the following form:

$$
P\left(\vec{a} \lambda_{a} ; \vec{b} \lambda_{b}\right)=P\left(\vec{a} \lambda_{a} \mid \vec{b} \lambda_{b}\right) P\left(\vec{b} \lambda_{b}\right) .
$$

The distribution $P\left(\vec{a} \lambda_{a} \mid \vec{b} \lambda_{b}\right)$ is the conditional of the event $\lambda_{a}$ ("yes" or "no") to occur under the condition that $\lambda_{b}$ ("yes" or "no") has occurred and $P\left(\vec{b} \lambda_{b}\right)$ is the marginal of the joint distribution. In the following, for notational convenience, we will omit the polarization directions and denote these functions by $P(i \mid j)$ and $P(j)$, where the indexes $i$ and $j$ will denote 1 and 0 for a "yes" or a "no" outcome, respectively. From the properties of the EPR entangled state (11) and the definitions of the projection operators (8) it is easy to calculate all of these distribution functions. The single-folded distributions are:

$$
P(0)=\frac{2 s}{2 s+1} \quad \text { and } \quad P(1)=\frac{1}{2 s+1} .
$$

Note that they are local i.e., polarization independent. The nonlocal polarization dependent conditional distribution is given by the following matrix:

$$
P\left(\lambda_{a} \mid \lambda_{b}\right)=\left[\begin{array}{ll}
P(0 \mid 0) & P(0 \mid 1) \\
P(1 \mid 0) & P(1 \mid 1)
\end{array}\right],
$$

where

$$
P(0 \mid 0)=\frac{1}{2 s}\left(2 s-1+\left(\sin \frac{\alpha}{2}\right)^{4 s}\right) \text { and } P(0 \mid 1)=1-\left(\sin \frac{\alpha}{2}\right)^{4 s} .
$$

In these formulas $\alpha$ is the relative angle between the two unit vectors $\vec{a}$ and $\vec{b}(\cos \alpha=\vec{a} \cdot \vec{b})$. From these joint and conditional probabilities we obtain that the joint spin transmission function is:

$$
p(\vec{a} ; \vec{b})=P(1 \mid 1) P(1)=\frac{1}{2 s+1}\left(\sin \frac{\alpha}{2}\right)^{4 s} .
$$

From the Bayes analysis (9) we have the following sum rules fulfilled by the conditional probabilities:

$$
P(0 \mid 0)+P(1 \mid 0)=1 \quad \text { and } \quad P(0 \mid 1)+P(1 \mid 1)=1 .
$$


This result shows that one can regard the EPR correlations as correlations of two sequences of random numbers represented by transmission functions $t\left(\vec{a}, \lambda_{a}\right)=\lambda_{a}$ and $t\left(\vec{b}, \lambda_{b}\right)=\lambda_{b}$ that are jumping between values 0 and 1 ("no" and "yes" answers) for measurements performed with linear analyzers. These are the only possible outcomes of the transmission experiment. This positive and nonlocal distribution leads to a simple statistical interpretation of the spin correlations and of the violation of Bell's inequality in terms of random numbers 1 and 0 for the transmission functions. On each single polarizer, the outcomes are completely random and the "yes" and "no" answers occur with probabilities $P(0)$ for "no" and $P(1)$ for "yes". The nonlocality of the EPR correlations shows up in the fact that these two perfectly random sequences (on the first and the second polarizers) are correlated and the correlations are given by Eq. (12). These formulas predict that the EPR entanglement can be understood as a nonlocal correlation between the two random sequences $t\left(\vec{a}, \lambda_{a}\right)=(1,0,0,1,1,0,0, \ldots)$ and $t\left(\vec{b}, \lambda_{b}\right)=(0,0,1,1,0, \ldots)$. The nonlocality of these correlations follows from the fact that whenever $t\left(\vec{b}, \lambda_{b}\right)=1$ on the polarizer $b$, we must have $t\left(\vec{a}, \lambda_{a}\right)=1$ or 0 on the polarizer $a$ with the probabilities $P(1 \mid 1)$ and $P(0 \mid 1)$, i.e., the outcomes on $a$ (possibly even a remote analyzer) are determined by the outcomes on the analyzer $b$. This is how the EPR quantum sequences of random numbers violate local realism. Only for $\alpha=0$ and $\alpha=\pi$ we have $P(0 \mid 0)=0$ and $P(1 \mid 1)=1$ and the events do occur with certainty. In this case the EPR correlations are perfect and the Bell's inequality is not violated. In Figure 1 and Figure 2 are plots of the conditional probabilities $P(1 \mid 0)$ and $P(0 \mid 1)$ as functions of the angle $\alpha$ for different values of the spin $s$. It is clear from these figures that with the increased value of the spin $s$, the probability $P(1 \mid 0)$ tends to zero while the probability $P(0 \mid 1)$ tends to one except for the angle $\alpha=\pi$, when $P(0 \mid 1)=0$. In this limit the random and nonlocal character of quantum mechanics goes away, and if $s \rightarrow \infty$ we have:

$$
P\left(\lambda_{a} \mid \lambda_{b}\right)=\left[\begin{array}{ll}
1 & 1 \\
0 & 0
\end{array}\right] \quad \text { for } \quad \alpha \neq \pi,
$$

and 


$$
P\left(\lambda_{a} \mid \lambda_{b}\right)=\left[\begin{array}{ll}
1 & 0 \\
0 & 1
\end{array}\right] \quad \text { for } \quad \alpha=\pi .
$$

This means that for $\alpha=\pi$ we have a $100 \%$ confidence that the outcome on $a$ will be the same as the outcome on $b$ and for $\alpha=0$ we now have $0 \%$ confidence that the outcomes on $a$ will be the same as the outcomes on $b$. This is precisely what we can expect from the entangled correlations in the classical limit corresponding in this case to $s \rightarrow \infty$. In this limit the quantum nonlocal distribution becomes a local probability distribution corresponding to sharp "yes" and "no" outcomes of the measurement involving classical antiparallel angular momenta. In quantum mechanic terms, in this limit the quantum amplitudes become classical probabilities for a classical spining top [11,12].

\section{B. RANDOMNESS AND INFORMATION}

We have shown that the outcomes of the EPR correlations can be visualized as nonlocal correlations between completely random sequences of random numbers $\lambda_{a}$ and $\lambda_{b}$. The transitions between these random numbers are described by joint and conditional probabilities given by Eq. (12). We have shown that due to the nonlocal character of these transition

probabilities the local reality of the spin correlations is incompatible with the quantum mechanical predictions. The nonlocality of the transition probabilities (12) violates the Bell's inequality.

On the other hand random sequences $\lambda_{a}$ and $\lambda_{b}$ with their correlations provide information about the correlated system of two spins. The joint or the conditional probabilities for these random sequences reflect what we know about the correlated system. This knowledge called information, can be described by the Gibbs-Shannon information-entropy H (in bits). From the definition of joint and marginal distribution functions we can construct the following information entropies for the EPR correlations

$$
H(a ; b)=-\sum_{\lambda_{a} \lambda_{b}} p\left(\lambda_{a} \vec{a} ; \lambda_{b} \vec{b}\right) \log _{2} p\left(\lambda_{a} \vec{a} ; \lambda_{b} \vec{b}\right)
$$

and its marginals: 


$$
H(\vec{a})=-\sum_{\lambda_{a}} p\left(\vec{a} \lambda_{a}\right) \log _{2} p\left(\vec{a} \lambda_{a}\right), H(\vec{b})=-\sum_{\lambda_{b}} p\left(\vec{b} \lambda_{b}\right) \log _{2} p\left(\vec{b} \lambda_{b}\right) .
$$

In the same way we introduce a conditional information entropy by the following definition

$$
H(\vec{a} \mid \vec{b})=-\sum_{\lambda_{a} \lambda_{b}} p\left(\lambda_{a} \vec{a} ; \lambda_{b} \vec{b}\right) \log _{2} p\left(\lambda_{a} \vec{a} \mid \lambda_{b} \vec{b}\right) .
$$

From the Bayes's theorem (9) relating a conditional probability with a joint probability we obtain that

$$
H(\vec{a} ; \vec{b})=H(\vec{a} \mid \vec{b})+H(\vec{b})=H(\vec{b} \mid \vec{a})+H(\vec{a}) .
$$

From the properties of the joint and the conditional probabilities follow the following inequality:

$$
H(\vec{a} \mid \vec{b}) \leq H(\vec{a}) \leq H(\vec{a} ; \vec{b})
$$

This inequality has a simple interpretation that removing a condition never decreases the information carried by the system.

For a system involving two correlated subsystems the entropy can offer a measure of quantum correlation if the information each of the subsystems is compared with the total information of the composite system. A very important property of the information-entropy is its subadditivity. The subadditivity states that [13]:

$$
H(\vec{a} ; \vec{b}) \leq H(\vec{a})+H(\vec{b})
$$

i.e, that when forming marginals one loses the information about the correlations. Before discussing some applications of subadditivity, let us make some remarks about the monotonicity of entropy. Neither quantum-mechanically nor in classical physics is it true that $H(\vec{a}) \leq H(\vec{a} ; \vec{b})$. This failure of monotonicity is expressed by the Araki and Lieb (AL) triangle inequality [14]:

$$
|H(\vec{a})-H(\vec{b})| \leq H(\vec{a} ; \vec{b}) \leq H(\vec{a})+H(\vec{b}) .
$$

with the right-hand side representing the subadditivity of the entropy. 
From the EPR marginals (10) we obtain:

$$
H(\vec{a})=H(\vec{b})=-\frac{1}{2 s+1} \log _{2} \frac{(2 s)^{2 s}}{(2 s+1)^{2 s+1}},
$$

i.e., the information contained in the single spin measurements for the two subsystems is equal to one bit for $s=\frac{1}{2}$ and is zero if $s \rightarrow \infty$. This follows from the fact that for $s=\frac{1}{2}$ we have $P(0)=P(1)=\frac{1}{2}$ and for $s \rightarrow \infty$ we have $P(0)=1$ with certainty. With these marginal informations the AL inequality (23) takes the following form

$$
0 \leq H(\vec{a} ; \vec{b}) \leq-\frac{2}{2 s+1} \log _{2} \frac{(2 s)^{2 s}}{(2 s+1)^{2 s+1}}
$$

where the joint entropy for the information accumulated by the two polarizers $\vec{a}$ and $\vec{b}$ is a function of $\cos \alpha=\vec{a} \cdot \vec{b}$. Let us note that this entropy is different from the von Neumann definition involving $\operatorname{Tr}\{\rho \ln \rho\}$. The EPR combined state is a pure state and as a result the von Neumann entropy will be equal to zero. The Gibbs-Shannon entropy as defined by Eq. (19) is different. It involves statistical information associated with possible "states" of the EPR system, labeled by indices $\lambda_{a}$ and $\lambda_{b}$ and described by a transition probability $P\left(\lambda_{a} \mid \lambda_{b}\right)$ to find the system in $\lambda_{a}$ if the system has been in $\lambda_{b}$.

In Figure 3 and Figure 4 we have plotted the conditional $H(\vec{a} \mid \vec{b})$, the joint $H(\vec{a} ; \vec{b})$ and the single $H(\vec{a})$ folded information entropies as a function of the angle $\alpha$ for spin- $\frac{1}{2}$ (Figure 3 ) and spin-2 (Figure 4). We see that the AL inequality (25) and the inequality (21) are satisfied for all values of $\alpha$. From this plot we see that the additivity of entropy (i.e., $H(\vec{a} ; \vec{b})=H(\vec{a})+H(\vec{b}))$ holds only for $\alpha=\pi$, i.e., when $\vec{a}$ is antiparallel to $\vec{b}$. This can be easily understood on the basis of the transition probabilities. For this geometry the only nonvanishing joint probabilities are $P(1 \mid 1)=P(0 \mid 0)=1$ and as a result $P(1 ; 1)=P(1)$ and $P(0 ; 0)=P(0)$. For the EPR correlations we conclude that the information satisfies the monotonicity condition, i.e., $H(\vec{a}) \leq H(\vec{a} ; \vec{b})$. Because of this property the AL inequality and the curves from the Figure 3 and the Figure 4 exhibit the subadditivity of the Gibbs-Shannon entropy when applied to the nonlocal transition probabilities $P\left(\lambda_{a} \mid \lambda_{b}\right)$.

Based on objective local realism, Braunstein and Caves (BC) [15, 16] have derived the 
information Bell's inequality involving the conditional entropies for pairs of probabilities. The BC inequality has the form:

$$
H(\vec{a} \mid \vec{b}) \leq H\left(\vec{a} \mid \vec{b}^{\prime}\right)+H\left(\vec{a}^{\prime} \mid \vec{b}^{\prime}\right)+H\left(\vec{a}^{\prime} \mid \vec{b}\right)
$$

and has the following simple physical interpretation, that four objective quantities cannot carry less information than any two of them. We shall apply this inequality to the information entropy that corresponds to the joint and conditional probabilities (12) predicted by quantum mechanics for the EPR correlations. For a coplanar geometry $a \cdot \overrightarrow{b^{\prime}}=\vec{a}^{\prime} \cdot \vec{b}^{\prime}=\vec{a}^{\prime} \cdot \vec{b}=\cos \alpha$ and $\cos 3 \alpha=\vec{a} \cdot \vec{b}$ the BC inequality (26) takes a simpler form:

$$
H(3 \alpha)-3 H(\alpha) \leq 0
$$

where by $H(\alpha)=H\left(\vec{a} \mid \vec{b}^{\prime}\right)$ we have denoted the conditional entropy as a function of the angle $\alpha$. In Figure 5 we have plotted the information difference from the left hand side of this inequality for different spins $s$. From these curves we conclude that the information Bell's inequality (26) as presented by BC is violated and that the violations decrease with the spin value $s$. Note that the degree of violation and the behavior of this violation for large values of the spin is different from the conclusions reached in [15]. This is due to the fact that in this reference the information entropy has been calculated for measurements which resolve consecutive values of $m$, and such measurements are inherently nonclassical in nature. The measurements presented in this paper and represented buy the projections (8) do not resolve values of $m$ and as a result do have a well defined classical limit.

\section{ENTANGLED GHZ CORRELATIONS}

\section{A. RANDOMNESS AND NONLOCALITY}

One particularly simple entangled GHZ state of three spin- $\frac{1}{2}$ particles, named $a, b$, and $c$ discussed by Mermin [17] has the following form: 


$$
\left|\psi_{\mathrm{GHZ}}\right\rangle=\frac{1}{\sqrt{2}}(|+,+,+\rangle-|-,-,-\rangle),
$$

where $|+\rangle$ or $|-\rangle$ specifies spin up or down along the appropriate $z$-axis. As showed by Mermin this entangled state provides an always versus never test of local realism. The joint probability for detection of the three particles $a, b$, and $c$ by three polarizers 1,2 and 3 , respective in an $\mathrm{x}-\mathrm{y}$ plane perpendicular to the particles' line of flight is:

$$
\begin{array}{r}
p(\phi)=p\left(\phi_{1} ; \phi_{2} ; \phi_{3}\right)=\left\langle\psi_{\mathrm{GHZ}}\left|\hat{P}\left(\phi_{1}\right) \otimes \hat{P}\left(\phi_{2}\right) \otimes \hat{P}\left(\phi_{3}\right)\right| \psi_{\mathrm{GHZ}}\right\rangle \\
=\frac{1}{8}\left(1-\cos \left(\phi_{1}+\phi_{2}+\phi_{3}\right)\right),
\end{array}
$$

where $\phi_{1}, \phi_{2}$ and $\phi_{3}$ represent the orientation angles of the detectors and $\phi=\phi_{1}+\phi_{2}+\phi_{3}$. The case where a definite prediction of spin measurement is possible corresponds to $\phi_{1}+\phi_{2}+\phi_{3}=$ $\pi$ with the joint probability equal to $p(\phi=\pi)=\frac{1}{4}$ and to $\phi_{1}+\phi_{2}+\phi_{3}=0$ with the joint probability equal to $p(\phi=0)=0$. These two cases correspond to perfect correlations, i.e., to such correlations when by measuring two spins one can predict with certainty the outcome of the measurement involving the third spin. Contrary to the EPR correlations, the GHZ correlations exhibit strong nonlocal properties for perfect correlations. While for the two-particle EPR spin-singlet state, perfect correlations can be made compatible with a stochastic model of hidden-variable theory based on local realism, the three-particle GHZ perfect correlations offer a never versus always refutation of local realities. It has been show that the GHZ correlations can be analyzed in the context of cavity quantum electrodynamics, and that the joint three-particle probability can be measured, in principle, by using single photon detection [18].

Following the basic ideas of a theory based on local reality, the transmission of an arbitrary spin- $s$ through linear polarizers $\phi_{1}, \phi_{2}$ and $\phi_{3}$ are described by objective realities with hidden variables $\lambda_{a}, \lambda_{b}$ and $\lambda_{c}$. In a theory based on LHV, as in the EPR case (3), the three-particle GHZ correlations are given in the form of the following statistical averages of local realities:

$$
p\left(\phi_{1} ; \phi_{2} ; \phi_{3}\right)=\int d \lambda_{a} \int d \lambda_{b} \int d \lambda_{c} P\left(\lambda_{a} ; \lambda_{b} ; \lambda_{c}\right) t\left(\phi_{1}, \lambda_{a}\right) t\left(\phi_{2}, \lambda_{b}\right) t\left(\phi_{3}, \lambda_{c}\right),
$$


where the hidden parameters are randomly distributed with a positive and normalized distribution function. For such a local distribution of random variables the LHV transmission probability (30) evaluated for four different polarization axes $\phi_{1}, \phi_{1}^{\prime}, \phi_{2}, \phi_{2}^{\prime}$ and a fixed $\phi_{3}$ is restricted by the following simple generalization of the Bell's inequality (5)

$$
\begin{array}{r}
-p\left(\phi_{3}\right) \leq p\left(\phi_{1} ; \phi_{2} ; \phi_{3}\right)+p\left(\phi_{1}^{\prime} ; \phi_{1} ; \phi_{3}\right)+p\left(\phi_{1} ; \phi_{2}^{\prime} ; \phi_{3}\right)-p\left(\phi_{1}^{\prime} ; \phi_{2}^{\prime} ; \phi_{3}\right) \\
-p\left(\phi_{1} ; \phi_{3}\right)-p\left(\phi_{2} ; \phi_{3}\right) \leq 0
\end{array}
$$

Using the spectral decomposition for the three GHZ projector operators the three-particle GHZ correlations can be written in the following form

$$
p(\phi)=\int d \lambda_{a} \int d \lambda_{b} \int d \lambda_{c} P\left(\phi_{1} \lambda_{a} ; \phi_{2} \lambda_{b} ; \phi_{3} \lambda_{c}\right) \lambda_{a} \lambda_{b} \lambda_{c}
$$

where the distribution function is defined as

$$
P\left(\phi_{1} \lambda_{a} ; \phi_{2} \lambda_{b} ; \phi_{3} \lambda_{c}\right)=\left\langle\psi_{\mathrm{GHZ}}\left|\delta\left(\lambda_{a}-\hat{P}\left(\phi_{1}\right)\right) \otimes \delta\left(\lambda_{b}-\hat{P}\left(\phi_{2}\right)\right) \otimes \delta\left(\lambda_{c}-\hat{P}\left(\phi_{3}\right)\right)\right| \psi_{\mathrm{GHZ}}\right\rangle .
$$

The relation of the randomness and the nonlocality of the GHZ correlations will be addressed with a statistical analysis of the three-particle distribution function. We discuss these correlations in the framework of Bayes analysis using the three-point generalization of the the two-point EPR (7) distribution [19]. For this distribution we can write:

$$
P\left(\phi_{1} \lambda_{a} ; \phi_{2} \lambda_{b} ; \phi_{3} \lambda_{c}\right)=P\left(\phi_{1} \lambda_{a} \mid \phi_{2} \lambda_{b} \phi_{3} \lambda_{c}\right) P\left(\phi_{2} \lambda_{b} \mid \phi_{3} \lambda_{c}\right) P\left(\phi_{3} \lambda_{c}\right)
$$

where the distribution $P\left(\phi_{2} \lambda_{b} \mid \phi_{3} \lambda_{c}\right)$ is the conditional of the event $\lambda_{b}$ (yes or no) to occur under the condition that $\lambda_{c}$ (yes or no) has occurred. The distribution $P\left(\phi_{1} \lambda_{a} \mid \phi_{2} \lambda_{b} \phi_{3} \lambda_{c}\right)$ is the conditional of the event $\lambda_{a}$ to occur under the condition that $\lambda_{b}$ and $\lambda_{c}$ have occurred. The distribution $P\left(\phi_{3} \lambda_{c}\right)$ is a one-fold marginal of Eq. (34). The three-particle distribution function is positive everywhere, but depends on the polarization directions $\phi_{i}$. The nonlocality of the GHZ distribution function makes the Bell's inequality (31) incompatible with quantum prediction. As in the previous section, for notational convenience, we will omit the polarization directions and denote these functions by $P(i \mid j k), P(i \mid j)$ and $P(j)$, where 
the indexes $i j$ and $k$ will denote 1 and 0 for a "yes" or "no" outcomes. From the GHZ spin state (28) we obtain that: $P\left(\lambda_{c}\right)=\frac{1}{2}$ and $P\left(\lambda_{b} \mid \lambda_{c}\right)=\frac{1}{2}$ for all values of $\lambda_{b}$ and $\lambda_{c}$ and all orientations of the polarizers. The the three-fold conditional probabilities are

$$
\begin{gathered}
P(1 \mid 11)=P(0 \mid 01)=P(1 \mid 00)=P(0 \mid 10)=\frac{1}{2}(1-\cos \phi), \\
P(0 \mid 00)=P(1 \mid 10)=P(1 \mid 01)=P(0 \mid 11)=\frac{1}{2}(1+\cos \phi)
\end{gathered}
$$

These last expressions reveal the statistical nature of GHZ correlations, that the value of the third spin is known with probability $\frac{1}{2}(1 \pm \cos \phi)$ if the other two spins have be measured. This statistical picture leads to a simple interpretation of GHZ correlations in terms of random numbers 1 and 0 . The quantum mechanical average in this case is represented by an ensemble average of three sequences of random numbers 1 and 0 , that are the only possible outcomes of the experiment. On each single polarizer or pair of polarizers the outcomes are completely random and the yes and no answers occur with equal probability $P\left(\lambda_{a} \mid \lambda_{b}\right)=\frac{1}{2}$. The GHZ correlations show that these completely random sequences are correlated if a third polarizer is involved. For example for $\phi=0$ if the two detectors have registered $(0,0)$ the third detector has to register the outcome 0 with certainty. For $\phi=\pi$, if the two detectors have registered $(1,1)$ the third detector has to register the outcome 1 with certainty. Any subsequence of random numbers composed only of pairs $\left(\lambda_{a}, \lambda_{b}\right)$, $\left(\lambda_{a}, \lambda_{c}\right)$ and $\left(\lambda_{b}, \lambda_{c}\right)$ is completely random and uncorrelated, while sequences involving three outcomes $\left(\lambda_{a}, \lambda_{b}, \lambda_{c}\right)$ are correlated and are described by a nonlocal-polarization-dependent distribution function. Note that the three-fold probability distribution (35) fails to satisfy the property that the probability of an event like the registration of each measurement depends only on the preceding outcome and not also on earlier states of the measured system. In short we have:

$$
P\left(\lambda_{a} \mid \lambda_{b} \lambda_{c}\right) \neq P\left(\lambda_{a} \mid \lambda_{b}\right) P\left(\lambda_{b} \mid \lambda_{c}\right)
$$

These conclusions are valid for any an arbitrary orientation of the polarizers and indicates that strong nonlocality is exhibited even for perfect correlations corresponding to $\cos \phi= \pm 1$. 


\section{B. RANDOMNESS AND INFORMATION}

The outcomes of the GHZ correlations can be visualized as nonlocal correlations between completely random sequences of random numbers $\lambda_{a}$ and $\lambda_{b}$ and $\lambda_{c}$. The transitions between these random numbers are described by joint and conditional probabilities given by Eq. (35). Due to the nonlocal character of these conditional probabilities the local reality of the GHZ spin correlations is incompatible with the quantum mechanical predictions. The nonlocality of the transition probabilities violates the Bell's inequality. On the other hand random sequences $\lambda_{a} \lambda_{b}$ and $\lambda_{c}$ with their correlations provide information about the correlated system of the three spins. The joint or the conditional probabilities for these random sequences reflect what we know about the GHZ correlated system. Following the definitions of the information entropy for the EPR correlations we introduce the following information entropy for the GHZ correlations:

$$
H\left(\phi_{1} ; \phi_{2} ; \phi_{3}\right)=-\sum_{\lambda_{a} \lambda_{b} \lambda_{c}} P\left(\phi_{1} \lambda_{a} ; \phi_{2} \lambda_{b} ; \phi_{3} \lambda_{c}\right) \log _{2} P\left(\phi_{1} \lambda_{a} ; \phi_{2} \lambda_{b} ; \phi_{3} \lambda_{c}\right)
$$

For a system involving three correlated subsystems the entropy can offer a measure of quantum correlation if the informations of the subsystems $\phi_{1} \cup \phi_{3}$ and $\phi_{2} \cup \phi_{3}$ are compared with the informations of the subsystems $\phi_{1}$ and $\phi_{3}$. For such measurements Lieb and Ruskai (LR) [20] have derived the following inequality:

$$
H\left(\phi_{1} ; \phi_{3}\right)-H\left(\phi_{1}\right)+H\left(\phi_{2} ; \phi_{3}\right)-H\left(\phi_{2}\right) \geq 0
$$

The LR inequality implies that more information can be obtained in joint measurements involving various two-particle correlations than in single measurement involving only one particle. For the GHZ correlations, $H\left(\phi_{1} ; \phi_{3}\right)=H\left(\phi_{2} ; \phi_{3}\right)=2$ and $H\left(\phi_{1}\right)=H\left(\phi_{2}\right)=1$ and we see that this inequality is satisfied independently of the polarization orientations. For the three-particle system the subadditivity for the entropy difference takes the following form:

$$
H\left(\phi_{1} ; \phi_{2} ; \phi_{3}\right)-H\left(\phi_{2}\right) \leq H\left(\phi_{1} ; \phi_{2}\right)-H\left(\phi_{2}\right)+H\left(\phi_{3} ; \phi_{2}\right)-H\left(\phi_{2}\right)
$$


This inequality implies that the information content of $\phi_{1} \cup \phi_{2}$ and $\phi_{3} \cup \phi_{2}$ relative to $\phi_{2}$ is greater than that of $\phi_{1} \cup \phi_{2} \cup \phi_{3}$ relative to $\phi_{2}$. For the GHZ wave function this inequality reduces to:

$$
H(\phi)=H\left(\phi_{1} ; \phi_{2} ; \phi_{3}\right) \leq 3
$$

As in the case of the LR inequality, this inequality is satisfied for all values of the the angle $\phi$. In Figure 6 we see a plot of the joint three-particle information entropy as a function of the polarizers angles forming $\phi$. We see that the the maximum information is reahced if $\phi_{1}+\phi_{2}+\phi_{3}=\pi$.

Based on objective local realism we generalize the BC information Bell's inequality (26) involving information entropies of the GHZ three particles. The three-particle BC information Bell's inequality for the GHZ correlations has the form

$$
H\left(\phi_{1} \mid \phi_{2} \phi_{3}\right) \leq H\left(\phi_{1} \mid \phi_{2}^{\prime} \phi_{3}\right)+H\left(\phi_{1}^{\prime} \mid \phi_{2}^{\prime} \phi_{3}\right)+H\left(\phi_{1}^{\prime} \mid \phi_{2} \phi_{3}\right)
$$

where $H(i \mid j k)$ are the conditional three-point distributions $P(i \mid j k)$ given by Eqs.(35). In

order to investigate the properties of this inequality, we select the following orientations of the polarization angles; $\phi_{1}^{\prime}=\frac{3 \pi}{4}, \phi_{2}^{\prime}=\frac{\pi}{2}, \phi_{1}=\frac{\pi}{4}$ and $\phi_{2}=0$ (see Figure 7 ). For this particular geometry the information Bell's inequality (41) for the GHZ correlations reduces to:

$$
H\left(\frac{\pi}{4}+\phi_{3}\right)-3 H\left(\frac{3 \pi}{4}+\phi_{3}\right) \leq
$$

In Figure 8 we have plotted the information difference represented by the left hand side of this inequality. From this curve we conclude that the information Bell's inequality (42) as presented by $\mathrm{BC}$ is violated.

\section{ACKNOWLEDGMENTS}

The author thanks C. Caves and G. Herling for numerous discussions and comments. This work was partially supported the Center for Advanced Studies of the University of New Mexico. 


\section{REFERENCES}

* Permanent Address

[1] For review see M. Redhead, Incompleteness Nonlocality and Realism (Clarendon, Oxford, 1987).

[2] A. Einstein, B. Podolsky, and N. Rosen, Phys. Rev. 47, 777 (1935).

[3] N. D. Mermin, Phys. Rev. D 22, 356 (1980).

[4] A. Garg and N. D. Mermin, Phys. Rev. Lett. 49, 901,1294 (E) (1982).

[5] N. D. Mermin and G. M. Schwarz Found. Phys. 12, 101 (1982).

[6] D. M. Greenberger, M. Horne, and A. Zeilinger in Bell's Theorem, Quantum Theory, and Conceptions of the Universe, edited by M. Kafatos (Kluwer Academic, Dordrecht, the Netherlands, 1989)

[7] D. M. Greenberger, M. A. Horne, A. Shimony, and A. Zeilinger, Am. J. Phys. 58, 1131 (1990).

[8] J. Bell, Physics 1, 195 (1964) ; J. F. Clauser and M. A. Horne, Phys. Rev. D10, 526 (1974).

[9] K. Wódkiewicz in Santa Fe Workshop of Foundations of Quantum Mechanics, eds. T. D. Black et al. (World Scientific, 1992) p. 276.

[10] K. Wódkiewicz and J. H. Eberly, Ann. Phys. (New York), 216, 268 (1992).

[11] K. Wódkiewicz, "Classical Limit of Entangled Correlations" Acta Phys. Pol. 86, 223 (1994).

[12] K. Wódkiewicz Phys. Rev. A 51, 2785 (1995).

[13] A. Wehrl Rev. Mod. Phys. 50, 221 (1978).

[14] H. Araki and E. H. Lieb Commun. Math. Phys. 18, 160 (1970) 
[15] S. Braunstein and C. M. Caves Phys. Rev. Lett. 61, 662 (1988).

[16] S. Braunstein and C. M. Caves Ann. Phys. (NY) 202, 22 (1990).

[17] N. D. Mermin, Am. J. Phys. 58, 731 (1990); Phys. Today 43 (6), 9 (1990).

[18] K. Wódkiewicz, Liwei Wang, and J. H. Eberly, Phys. Rev A 47,3280 (1993)

[19] K. Wódkiewicz, Liwei Wang, and J. H. Eberly, in Fundamentals of Quantum Optics III ed. F. Ehlotzky (Springer, 1993) p. 306.

[20] E. H. Lieb and M. B. Ruskai J. Math. Phys. 14, 1938 (1973) 


\section{FIGURES}

FIG. 1. The conditional distribution function $P(1 \mid 0)$ as a function of the angle $\alpha$ for different vaules of the spin. Spin- $\frac{1}{2}$ curve (a), spin-1 curve (b), spin-2 curve (c), spin-5 curve (d).

FIG. 2. The conditional distribution function $P(0 \mid 1)$ as a function of the angle $\alpha$ for different vaules of the spin. Spin- $\frac{1}{2}$ curve (a), spin-1 curve (b), spin-2 curve (c), spin-5 curve (d).

FIG. 3. Plots of the information entropies $H(\vec{a} \mid \vec{b})$ curve (a), $H(\vec{a})$ curve (b) and $H(\vec{a} ; \vec{b})$ curve (c) as functions of the angle $\alpha$ for $\operatorname{spin} s=\frac{1}{2}$.

FIG. 4. Plots of the information entropies $H(\vec{a} \mid \vec{b})$ curve (a), $H(\vec{a})$ curve (b) and $H(\vec{a} ; \vec{b})$ curve (c) as functions of the angle $\alpha$ for spin $s=2$.

FIG. 5. Plots of the information difference in the BC Bell's inequality as functions of the angle

$\alpha$ for for different values of the spin. Spin- $\frac{1}{2}$ curve (a), spin-1 curve (b), spin-2 curve (c), spin- 5 curve (d).

FIG. 6. Plot of the three-particle GHZ information as a function of the polarizers angle $\phi$.

FIG. 7. The coplanar orientations of the three polarizers with $\phi_{1}^{\prime}=\frac{3 \pi}{4}, \phi_{2}^{\prime}=\frac{\pi}{2}, \phi_{1}=\frac{\pi}{4}$ and $\phi_{2}=0$.

FIG. 8. Plot of the three-particle GHZ information difference following from the Bell's inequality as functions of the angle $\phi_{3}$. 


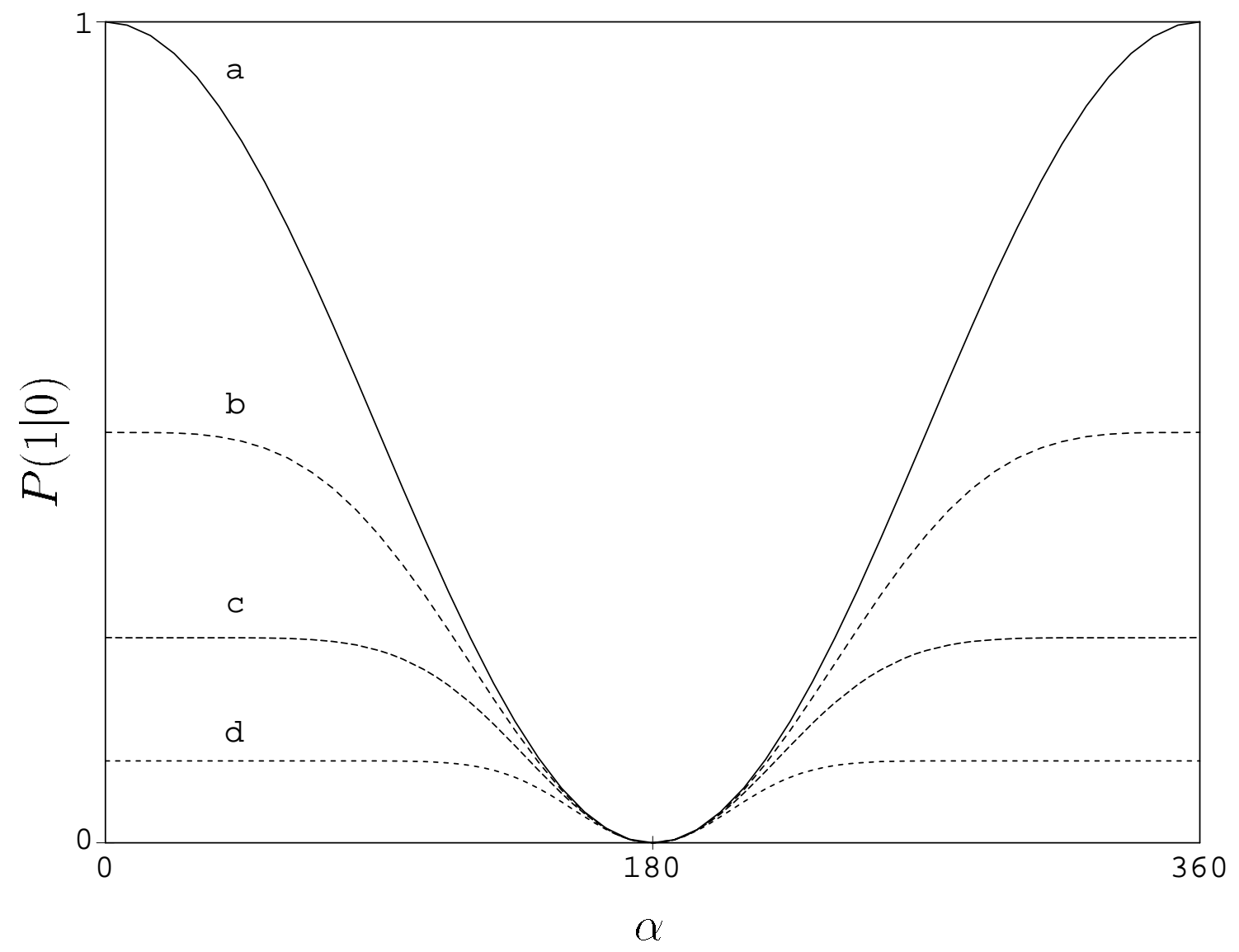

Wódkiewicz, Fig. 1 


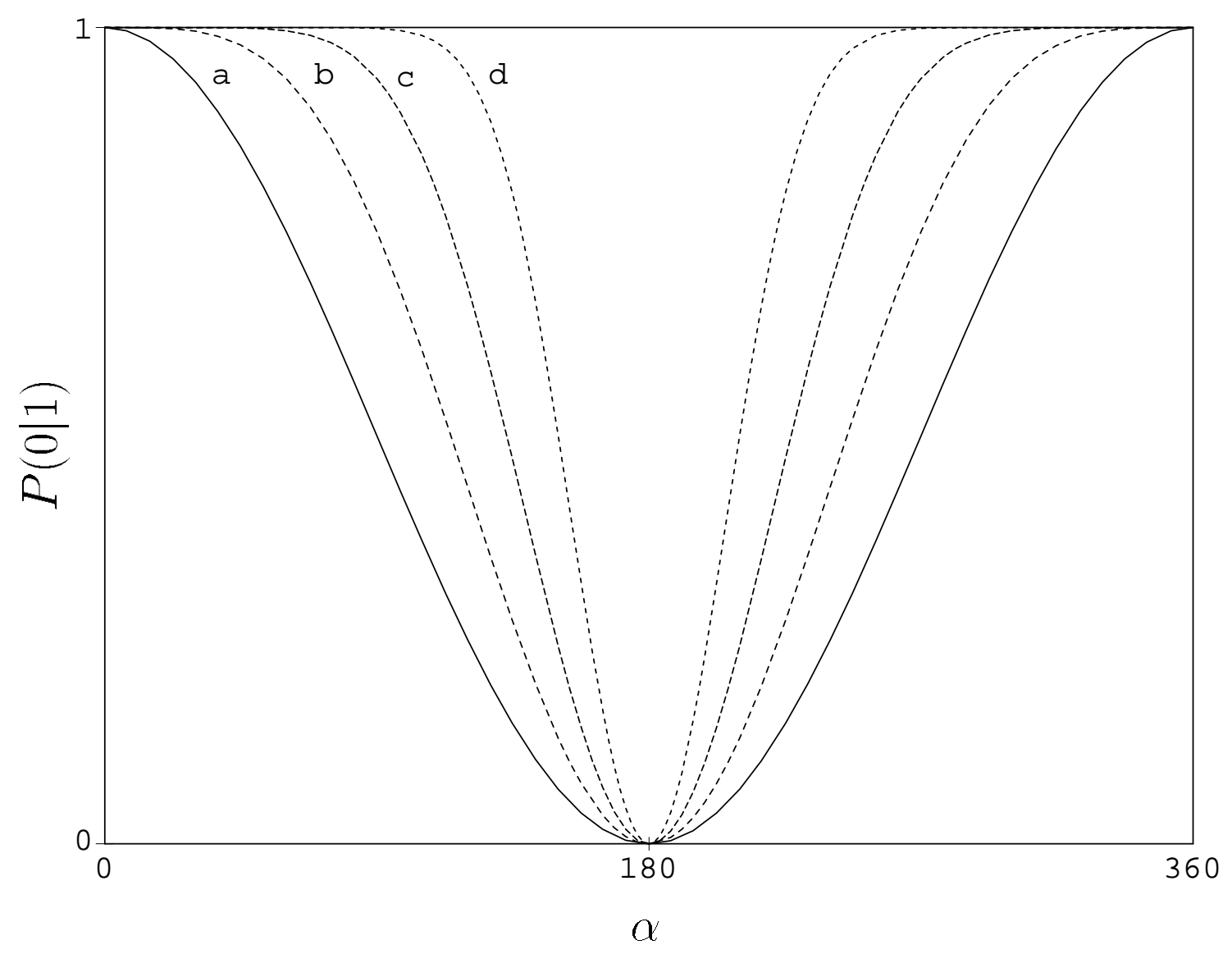

Wódkiewicz, Fig. 2 


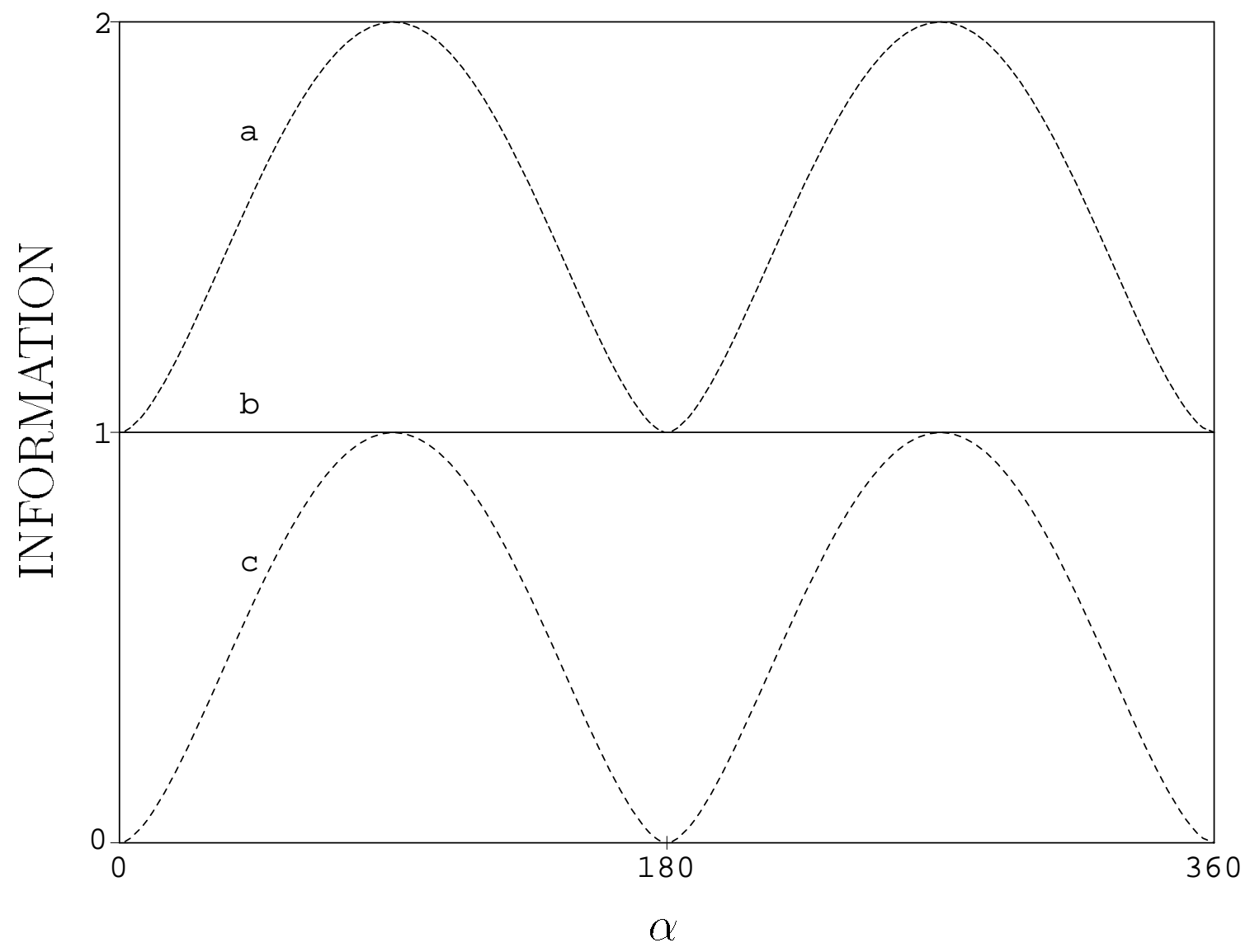

Wódkiewicz, Fig. 3 


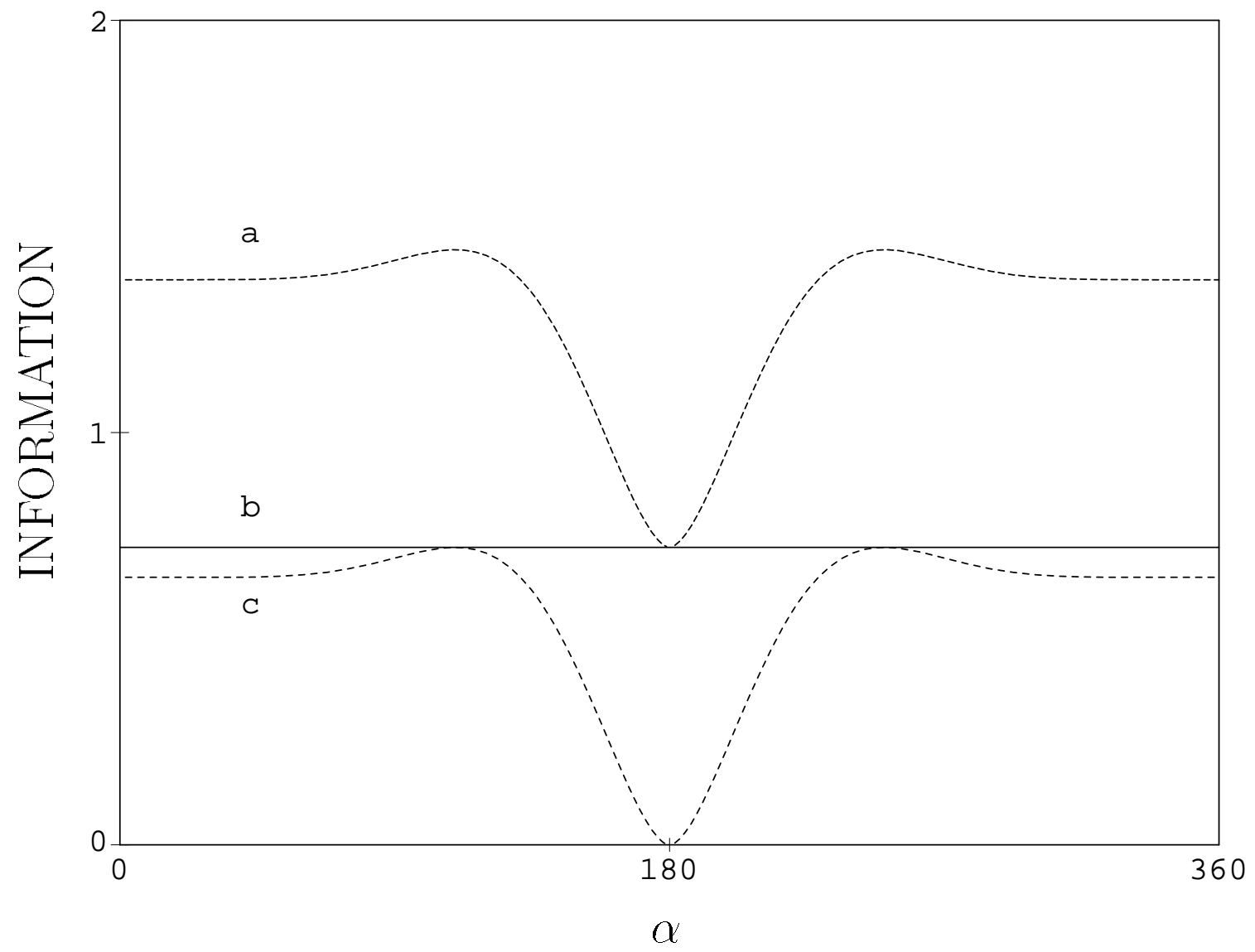

Wódkiewicz, Fig. 4 


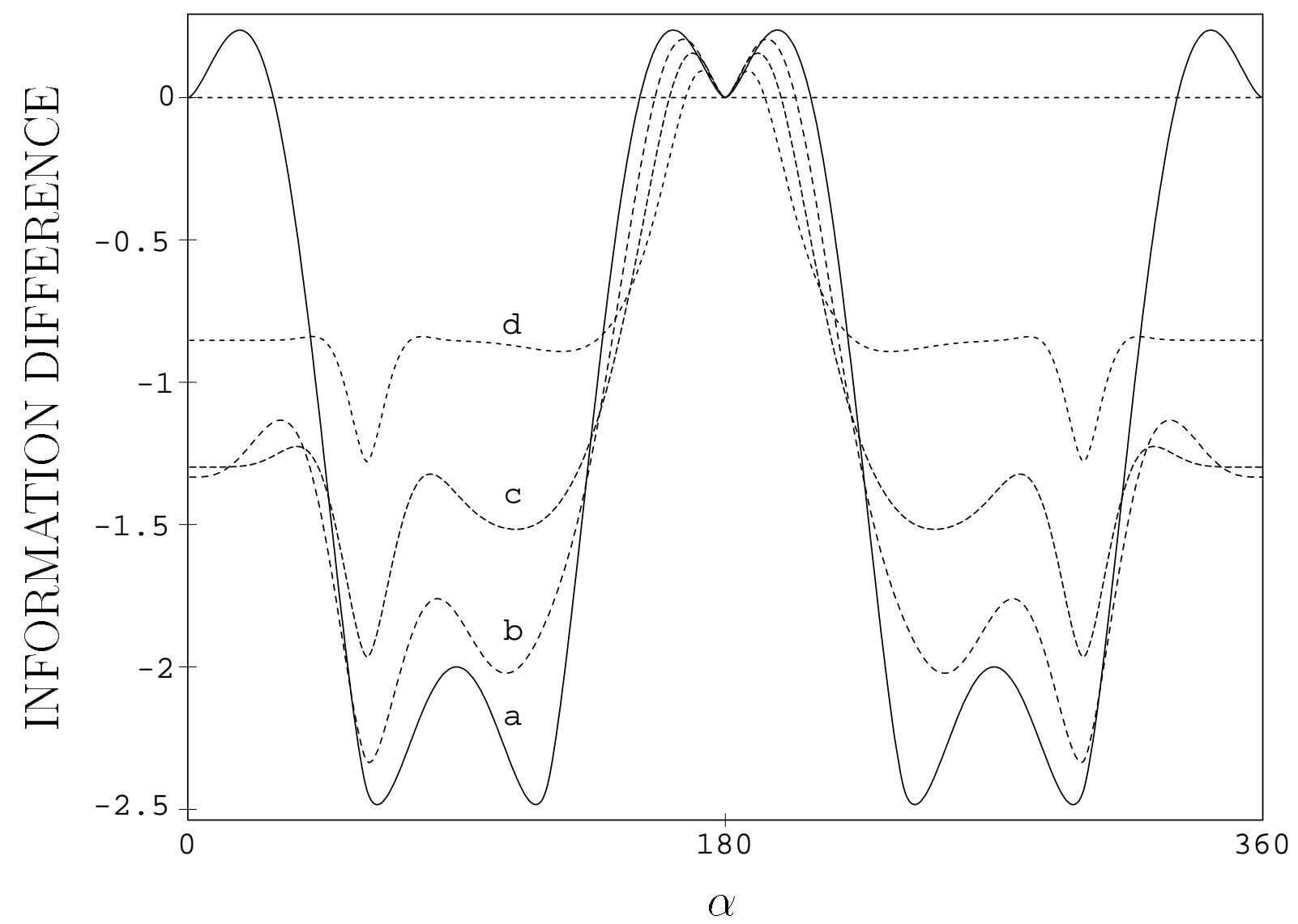

Wódkiewicz, Fig. 5 


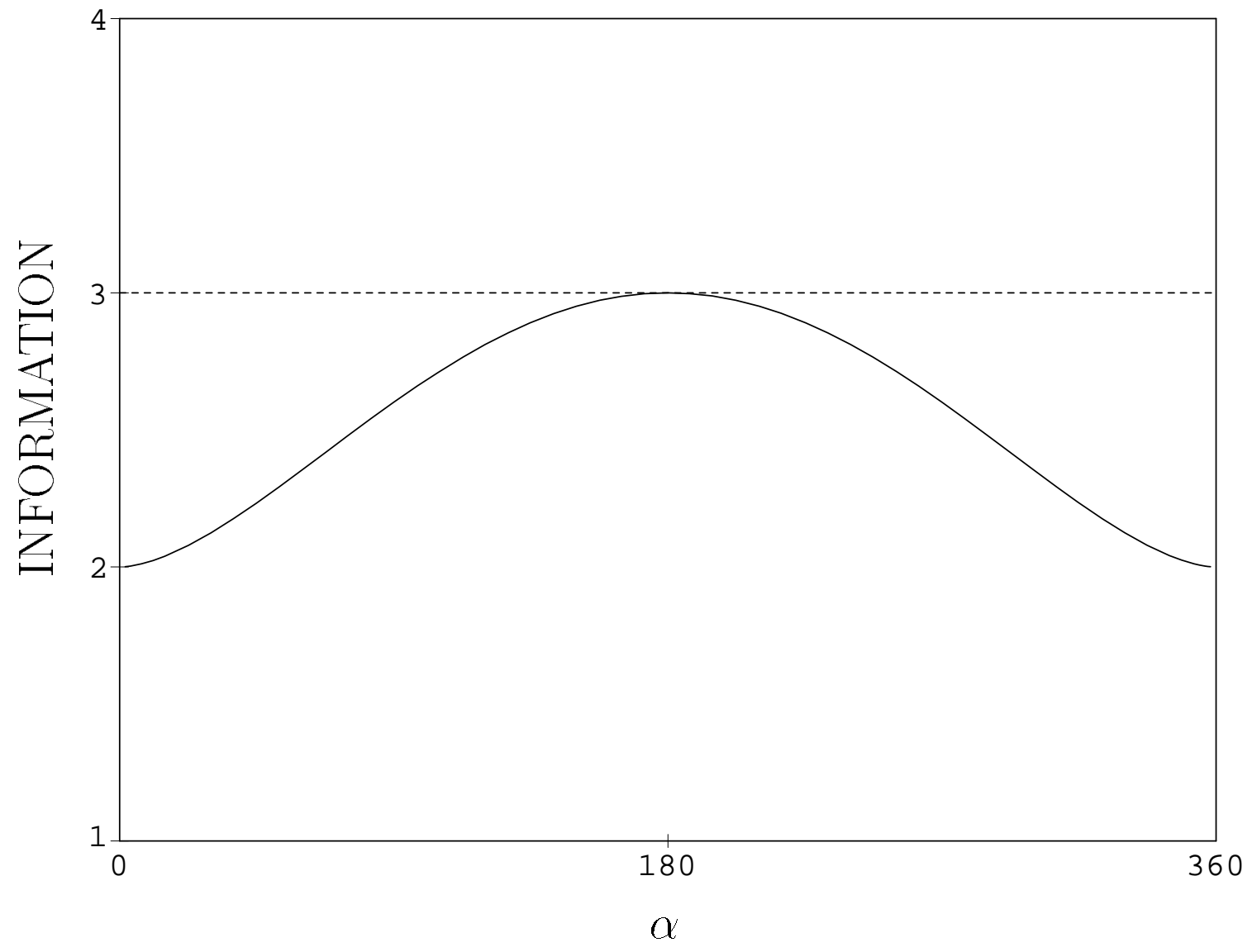

Wódkiewicz, Fig. 6 


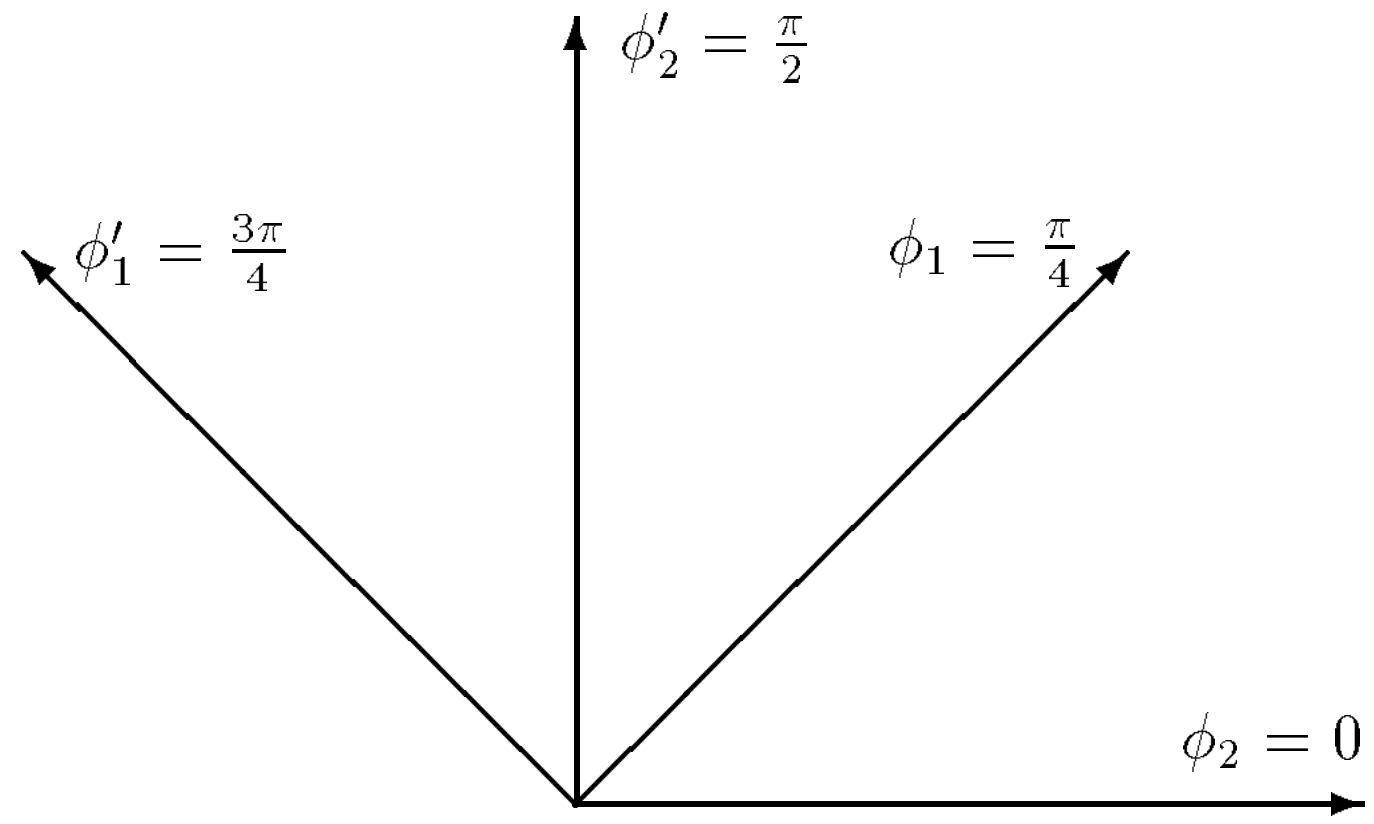

Wódkiewicz, Fig. 7 


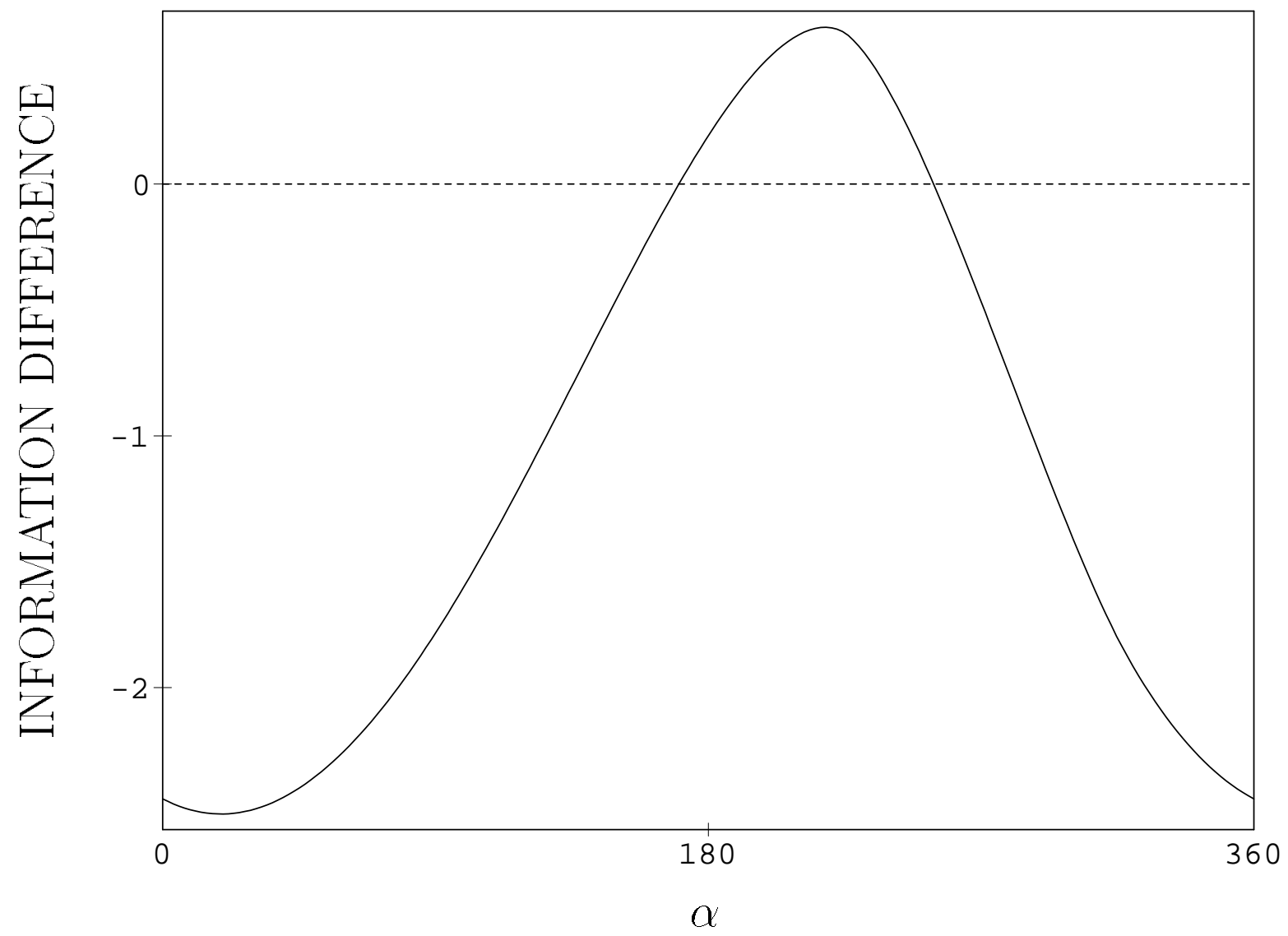

Wódkiewicz, Fig. 8 